\title{
An alternative approach to unifying chemistry with quantum mechanics
}

\author{
Vanessa A. Seifert ${ }^{1}$
}

Published online: 20 May 2017

(C) The Author(s) 2017. This article is an open access publication

\begin{abstract}
Harold Kincaid in Individualism and the Unity of Science postulates a model of unity-without-reduction in order to accurately describe the relation between individualism and macroeconomics. I present this model and apply it to the description of the relation between chemistry and quantum mechanics. I argue that, when it comes to the description of molecular structure, chemistry and quantum mechanics are unified in Kincaid's sense. Specifically, the two disciplines contribute to the formation of a unified body of knowledge with respect to molecular structure.
\end{abstract}

Keywords Unity $\cdot$ Supervenience $\cdot$ Quantum mechanics

\section{Introduction}

The relation between chemistry and quantum mechanics is extensively debated ${ }^{1}$ in the philosophy of chemistry. This is reasonable, considering the still vibrant debate in the philosophy of science about the relation between sciences in general [for example Curd and Cover (1998), Kellert et al. (2006), Dizadji-Bahmani et al. (2010), Bokulich (2008)].

The different positions presented in the philosophy of chemistry literature on this topic, could be understood as forming a range of proposals that reside within two extremes. At one extreme, chemistry is taken to be reduced to quantum mechanics so much so that, at least in principle, quantum mechanics could substitute chemistry in the description,

1 For example Gavroglu and Simoes (2012), Hendry (2004, 2006b, 2010), Hendry et al. (2012), Hettema (2014), Lombardi and Labarca (2005), Lombardi (2014), Needham (2010), Scerri (2012), Scerri and Fisher (2016), Schummer (2014), Le Poidevin (2005), Ramsey (1997).

Vanessa A. Seifert

vs14902@bristol.ac.uk

1 Department of Philosophy, University of Bristol, Cotham House, Bristol, UK 
explanation and prediction of chemical phenomena (for a view that is around these lines see Hettema 2012). At the other extreme, looking for a connection between the two disciplines is not meaningful or useful since the research groups that employ chemical or quantum mechanical descriptions have different research goals, employ different methods, and develop substantially different or incompatible conceptions (for example Schummer 2014). ${ }^{2}$

The paper presents a novel position about the relation of chemistry and quantum mechanics, that resides within the two extremes. Specifically, it presents Kincaid's model of unity and examines whether it applies for the case of chemistry's and quantum mechanics' descriptions of molecular structure. The resulting conclusion is that chemistry and quantum mechanics form a "integrated and interleveled" (Kincaid 1997) body of knowledge about molecular structure. ${ }^{3}$

\section{Sketching the content of the two disciplines}

Before presenting Kincaid's model, some initial clarifications are made. Firstly, the paper doesn't use the term 'theory' when referring to chemistry and quantum mechanics. Instead, it refers to them as 'disciplines'. Let me explicate why. Chemistry and quantum mechanics involve the development of theoretical postulations and conceptions in order to describe, explain and predict a particular domain of phenomena. Often, they specify, support or explain those postulations via the use of mathematical models, visual representations, experimental or semi-empirical methods. This aspect should not be excluded from a discussion of their relation. Firstly, because neglecting this would provide a distorted image of how chemistry's and quantum mechanics' descriptions are formulated, and explained. Secondly, because it would exclude important aspects of Kincaid's model of unification; namely of a unification that depends on the confirmatory, explanatory and heuristic interaction of the two disciplines. In my view, the term 'discipline' captures more accurately the fact that models, representations etc. are taken into account when examining the relation between chemistry and quantum mechanics.

Furthermore, the paper argues that if chemistry's and quantum mechanics' relation complies to Kincaid's model, then their respective descriptions of molecular structure can be understood as forming a unified 'body of knowledge'. 'Body of knowledge' refers not only to the theoretical postulations and conceptions developed for the prediction and explanation of a specific phenomenon. It also includes the mathematical models, visual representations, approximations, semi-empirical methods and experimentations that are involved in the description of that phenomenon.

Moreover, let me specify the main concepts postulated for the descriptions of molecular structure, by reference to the two disciplines' scales. 'Scale' refers to the particular time, length or energy scale to which the objects of a discipline are relative. For example, at "the

\footnotetext{
${ }^{2}$ There are also pluralist and emergentist understandings of chemistry that aren't restricted to its methodological or conceptual incompatibility with quantum mechanics. See for example Chang (2012), Hendry (2006b), Lombardi and Labarca (2005).

3 Whether this sort of unification applies to descriptions of other chemical phenomena could possibly be argued for, but isn't pursued here.

4 Kincaid employs the term 'theory' when presenting his model of unity-without-reduction. Also, the term 'body of knowledge' is an added element that is proposed here. The paper slightly differentiates itself from Kincaid's original unificatory model, without however distorting its core thesis.
} 
quantum scale there are no cats; at scales appropriate for astrophysics there are no mountains (..)" (p. 199, Ladyman and Ross 2007).

In chemistry, the time and length scale within which the chemical objects are defined is not particularly fine grained. Chemical phenomena are described with reference to objects of substantially different time and length scales; atoms, molecules and electrons are relative to a different time and length scale compared to, say, grams of iron reacting with oxygen gas. Since the paper is concerned with the description of molecular structure, it only focuses on the former scale.

Specifically, chemistry tracks molecular structure by reference to molecules, atoms, chemical bonds, electrons and nuclei. The structure of the molecule is described in terms of the characterisation of the atoms that make it up, and in terms of the interactions that take place between them (namely via the chemical bond). In quantum mechanics, the basic entities employed are nuclei and electrons. The Schrödinger equation describes the movement and energies of each of the participating nuclei and electrons and then, by employing approximate models, one can calculate the behaviour of the aggregate that they form.

Two points are worth specifying based on the above. First, chemistry and quantum mechanics describe molecular structure in partially overlapping (thus distinct) scales. Second, although certain entities belong to the scale of both disciplines (i.e. electrons and nuclei), those entities have different epistemic significance in the two descriptions. Chemistry refers to electrons and nuclei in order to explain molecular structure, but does not provide a complete descriptive and explanatory account of those entities. On the other hand, quantum mechanics is in the business of explaining and describing electrons and nuclei. In fact, while electrons and nuclei are employed in chemical descriptions of molecular structure, they are taken in the context of their characterisation in quantum descriptions. Chemistry employs descriptions of atomic structure in order to describe and explain molecular structure, and as such, those descriptions are taken as already established postulations that have been supported in quantum mechanics. ${ }^{5}$

The next section presents the seven criteria that make up Kincaid's model of unity, and argues that they hold with respect to the two disciplines' descriptions of molecular structure.

\section{The seven elements of Kincaid's unity}

Kincaid proposed a non-reductive model in order to argue for the unification of individualism and macroeconomics. Interestingly, a similar unificatory view was held by Pierre Duhem for the relation of chemistry to physics (Needham 2010). Although Duhem did not extensively develop the requirements of such a unification, Duhem's unity is based on a non-reductive, heuristic and explanatory interdependence between the two sciences (p. 166 Needham 2010); something that Kincaid explicitly requires in order for his model to hold.

The model is defined via seven elements ${ }^{6}$ which, if they hold with respect to the two descriptions of molecular structure, establish unity and support the existence of a unified

\footnotetext{
5 Although the determination of chemical and quantum scales implies some sort of integration of quantum mechanical postulates to chemical descriptions, I don't elaborate on this point until Kincaid's model is fully presented.

6 The model also explicitly rejects reducibility and strong emergence. This aspect of the model isn't currently examined due to limited word length.
} 
body of knowledge about molecular structure. These elements define unification in Kincaid's sense, but also act as criteria for this unification to hold. According to Kincaid, the first three elements (namely (1), (2), (3) below) suffice for unity to hold. If it is possible to support the other four criteria as well (namely (4), (5), (6), (7) below), then the position is more strongly defended. Let us examine each element in detail.

(1) The higher-level entities, postulated by chemistry for the description of molecular structure, are either composed of, or token identical to the entities postulated by the quantum mechanical description of molecular structure. (p. 66, Kincaid 1997)

The paper's interest lies in the relation of the two disciplines with respect to their descriptions of molecular structure. In this context, it is warranted to restrict the examination of the first criterion only to the higher level entities that are involved in the chemical description of molecular structure; namely atoms, molecules and chemical bonds.

Firstly, some clarifications about the main terms need to be stated. By the term 'entity' the paper doesn't include properties ${ }^{7}$; namely attributes, characteristics, features or qualities that can be postulated or attributed to things (Orilia and Swoyer 2016). Chemical properties that are involved in the description of molecular structure such as bond energy, bond length etc. do not fall under Kincaid's first criterion but are addressed within the third criterion (namely (3)).

Also, by 'composed' the paper refers to a part-whole relation ${ }^{8}$ between entities. This relation is not merely an aggregative relation, in the sense of a thousand grains of sand composing a heap. Rather, it also includes the possibility that the whole is partially determined by the physical interactions ${ }^{9}$ between those parts. In other words, composition is not restricted to a material, aggregative composition of the parts; it includes their respective physical interactions as well.

Consider then the atom. The atom is the most basic constitutive unit in chemistry. It is understood as comprised of a nucleus (i.e. a number of protons and neutrons) and of a number of negatively charged electrons that surround it. The entities that compose chemistry's atom (i.e. protons, neutrons and electrons), are the lower-level entities that are postulated by quantum mechanics. The manner in which lower level entities make up the atom according to quantum mechanics, is fully accepted by chemistry. For example, chemistry accepts that electrons move in a wave-like manner around the nucleus and that, due to the pull of the nucleus, electrons are confined to have particular energies (i.e. to be quantised), and to move within certain boundaries around the nucleus.

The aforementioned image of the atom has direct consequence for chemistry's description of molecules. A molecule represents a group of atoms that are arranged in a particular manner in space and are held together via chemical bonds. Therefore, based on the image of the atom, the molecule is ultimately composed of quantum mechanical entities as well; namely nuclei and electrons.

\footnotetext{
7 This is a plausible interpretation of Kincaid's requirement about 'entities', since Kincaid presents a separate criterion that pertains to 'properties' (i.e. the requirement of supervenience in "Chemical properties supervene on quantum mechanical properties (p. 66, Kincaid 1997)" section).

8 The paper doesn't examine the philosophical debates concerning the nature of part-whole relations.

${ }^{9}$ Physical interactions are gravitational, electromagnetic, strong nuclear and weak nuclear interactions. In the case of the quantum mechanical description of molecules, the interactions with the largest effect are electromagnetic in nature, so the paper primarily refers to this type.
} 
Concerning the chemical bond, there has been extensive debate [for example Hendry (2006a), Weisberg (2008)] about whether it refers to a material part of a molecule or to a state of energetic stability of molecules. Specifically, the structural conception takes that chemical bonds are material parts that signify the spatial relationship between a pair of atomic nuclei within a molecule (p. 917, Hendry 2006a). The energetic conception takes chemical bonding to signify "facts about energy changes between molecular or supermolecular states." (p. 919, Hendry 2006a). The paper doesn't defend a particular conception of the chemical bond; rather it argues that under either of the two conceptions, Kincaid's criteria are satisfied.

Specifically, the structural conception of the chemical bond understands it as a material part of molecules and thus as a higher level entity. Therefore, for Kincaid's model to be correct, the (structural) chemical bond should be either composed of or token identical to lower-level entities. Indeed this is the case. In chemistry it is represented as being the material part within the molecule that links atomic centres through the interaction of each atom's outer shell electrons. Electrons are part of the structural chemical bond, in the sense that they contribute, via their mutual interaction, to the stability that is achieved between the participating atomic centres, and consequently, to the stability of the entire molecule. Quantum mechanics describes the structural chemical bond in terms of the wave function of the participating lower level entities which specifies their interaction and behaviour. In this context, the structural chemical bond is indeed composed of the electrons and their interactions, both between each other and with the respective nuclei (that make up the atomic centres in the chemical level). However, it should be noted that, at the quantum level, the structural chemical bond in not composed of electrons in an aggregative individualistic manner; rather they compose it in the sense that they are "occupancies of non arbitrary partitions of the full electronic wave function that can be associated with the bond" (p. 918, Hendry).

Concerning the energetic conception, even if the chemical bond refers to a set of energetic properties of the entire molecule, Kincaid's account is not challenged. This is because, the energetic chemical bond (understood as a property) is supervenient on quantum mechanical properties. This argument is elaborated in criterion (3).

(2) Chemistry and quantum mechanics are logically compatible (p. 66, Kincaid 1997)

Logical compatibility demands that chemistry and quantum mechanics do not make contradictory claims. In order to support the logical compatibility of chemistry and quantum mechanics, the paper makes a counterfactual claim and a socio-historical one. The counterfactual claim states that, if chemistry assumed forces that are not postulated by quantum mechanics, i.e. forces that are independent and different from the four main forces postulated in the totality of physics, then indeed, this would suffice to claim that chemistry is incompatible with quantum mechanics. Since however there are not such chemical forces (on the contrary the forces postulated in the explanation of chemical behaviour are all physical), then there is no such logical incompatibility.

This argument by itself does not suffice to support the logical compatibility of chemistry and quantum mechanics. However, it acts as a positive indication in favour of the logical compatibility of the two disciplines. Proceeding in a similar manner by examining all other possible counterfactual claims will prove impractical and perhaps even untenable. Therefore, I support the claim by invoking the following socio-historical argument.

The development of quantum chemistry ${ }^{10}$ does imply that the two disciplines must be logically compatible, for if they weren't, firstly, such a sub-discipline would not exist, and

${ }^{10}$ Quantum chemistry is the sub discipline of chemistry that applies quantum models for the description, prediction and explanation of chemical phenomena. 
secondly, this would necessarily lead to the amendment or rejection of one of the two theories, or at least to the recognition of an anomaly in one of them. ${ }^{11}$ This is supported by the fact that in cases where the chemical description was incomplete or implicitly promoting an incompatible image with respect to quantum mechanics, chemistry has revised its postulations and incorporated quantum mechanics' main theses. ${ }^{12}$

The most striking example of the above is how chemistry's image of the atom significantly changed with the incorporation of quantum mechanics' postulations about the wave-like manner of entities, the quantisation of energy levels, and spin. I briefly present this example by focusing on three particular events.

Firstly, quantum mechanics' postulations of the wave-like manner of entities affected and influenced chemistry's image of the atom. The proposition that entities do not only behave as particles but also as waves had a direct effect on the way atoms were perceived and on how their inner structure could be described. In this context, Schrödinger formulated the second-order differential equation that would describe the behaviour of a particle in terms of its wave-like characteristics. Specifically, electron behaviour was described in terms of the Schrödinger equation which is the mathematical representation of the trajectory of electrons. Added to this is the de Broglie relation which calculates the wave length of the electrons and relates it to their momentum via Planck's constant, $h$.

Secondly, Planck's proposal that the amount of energy emitted from a blackbody in thermal equilibrium is discrete and not continuous, had a significant effect on the model of the atom as well. Specifically, Bohr incorporated Planck's conclusion (p. 262-269, Pullman 1998) in his model of the atom, by postulating stable electron orbits around the nucleus and by suggesting that, when an electron occupying a specific orbit 'jumps' onto another orbit, it either releases or absorbs a discrete amount of energy.

Thirdly, the Schrödinger equation brought in a natural way Bohr's image of the atom since it generated and specified Bohr's three quantum numbers (p. 277, Pullman 1998). Bohr's quantum numbers that were "associated with the size, shape, and spatial orientation" (p. 268, Pullman 1998) of the orbits, were now inferred by quantum mechanics instead of merely being postulated.

In conclusion, the aforementioned events illustrate that developments in quantum mechanics were not only accepted by chemistry but were incorporated in chemical descriptions and explanations of atomic and molecular behaviour. This reveals the success and continuous effort to preserve the logical compatibility of the two theories.

(3) Chemical properties supervene on quantum mechanical properties (p. 66, Kincaid 1997)

Kincaid understands supervenience in the sense that fixing a set of lower level properties fixes any higher level property (p. 72, Kincaid 1997). What Kincaid means by 'fix' is not entirely clear, so the paper assumes an understanding of supervenience that is compatible with Kincaid's model. The paper presents the basic points derived from Kincaid's understanding of supervenience and then provides a definition of the term that is compatible with Kincaid's understanding of supervenience.

\footnotetext{
${ }^{11}$ Logical compatibility should not be confused with other sorts of compatibilities, like conceptual or methodological compatibility. These incompatibilities could be argued for, however, they do not challenge the fact that the two disciplines are logically compatible. (For more on conceptual compatibility see Schummer 2014).

12 See Gavroglu and Simoes (2012) for an account of how the development of quantum mechanics affected chemistry and resulted to the formation of quantum chemistry.
} 
The basic points that constitute Kincaid's understanding of supervenience are the following;

I. Supervenience does not ensure the existence of bridge laws, identity relations or definitions that connect lower level with higher level properties. Specifically, it is not required that one is able to describe the specific relationship between chemical and quantum properties. Supervenience does not provide any systematic way of describing such relations, it merely postulates that some sort of relation between the quantum and chemical ontology exists.

II. It is possible for a lower level property to fix or determine more than one higher level property. In fact, it might be the case that the way higher and lower level properties cut up the world is very different, making it possible for a property of a higher level theory never to be equivalent to one or more properties of the lower level theory. Therefore, it is not required that a specific chemical property is related to a specific quantum property.

III. Supervenience is not refuted if the lower level predicates that describe lower level properties are derived through the use of ad hoc information from higher level predicates of the respective higher level description of that system. It is irrelevant whether the description of lower level entities and properties is facilitated by information provided from the description of the respective higher level entities and properties that are composed or supervenient upon them.

IV. It allows multiple realisability; namely it is possible for two systems to differ with respect to their lower level properties, while not differing with respect to their higher level properties. In terms of our discussion, this amounts to allowing the possibility of two entities differing with respect to some set of their quantum properties, while not differing with respect to their chemical properties. ${ }^{13}$

V. Supervenience is not taken to be a necessitation relation between higher and lower level properties; it is not the case that a change in the chemical properties of an entity necessitates a specific change in the quantum properties of that entity. It merely postulates that there is a change in quantum properties, when there is a change in chemical properties. Whether this is necessitation relation between higher and lower level properties, or a relation of a different modal force is not examined and our account of supervenience allows for different interpretations of that relation.

Supervenience then is an ontological position with regard to the relation between chemical and quantum mechanical properties. It postulates the existence of a relation between chemical and quantum mechanical properties, such that when any chemical property of a molecule (say its structure) changes, then it is always the case than one or more of the quantum mechanical properties of its composing (or token identical) lower level elements have changed as well. However, this does not entail, neither that this is a one-to-one relation, nor that such a relation be epistemically describable by the chemical or quantum formulation (see point I). More importantly, it doesn't have to be the case, for supervenience to hold, that one is able to pin point which specific set of properties as described in the quantum mechanical formalism correspond or (at least) change when a chemical property changes.

${ }^{13}$ Whether this is the case between chemical and quantum properties does not challenge our understanding of supervenience, so the paper doesn't examine whether multiple realisability holds. 
A sufficient, though not necessary, argument for supervenience would be to show that when the complete chemical description of a molecule changes, then its relevant complete quantum mechanical description changes as well. Notice that this sort of epistemic support of supervenience rests on the assumption that both descriptions are complete in their characterisation of a molecule. That is, it is not the case that either the chemical or the quantum mechanical description miss out in describing some chemical or quantum mechanical property, process or interaction that takes place within the system; the system is fully described within the context of the chemical and quantum mechanical formulations.

Although this is a possible strategy to follow in order to defend supervenience, it will not be elaborated here, mainly due to restrictions in the length of the paper. Instead, it is argued that rejecting supervenience would lead to conclusions that are not supported by the our best current chemistry and quantum mechanics.

Let us suppose then that supervenience does not hold. It follows that there is at least one higher level property of a system that, when it changes, there is no lower level property of the system that changes; the system remains invariant on the lower level. In this case, this would amount to claiming that when a chemical property of a system changes, none of its quantum mechanical properties change. Epistemically this would be reflected by the fact that the (complete) quantum mechanical description of the system remains the same as before the change in the chemical description.

Although supervenience is not here defined in terms of determination or realisation, from its rejection it follows that there are at least some aspects of the higher level behaviour of a system (i.e. property, interaction, process) that is not determined or realised by its lower level entities and their respective interactions; they are in principle inexplicable by any sort of description of its lower level entities and properties. For chemical properties this can be stated as followed: there is at least one chemical property of the system that is not determined by the existence, interactions and properties of its composing quantum parts (i.e. electrons and nuclei). It is not only that the change in a chemical property has not been detected in the quantum description; most importantly, such a change is ontologically undetermined by any lower level set of properties of its parts.

Do our best current physical and chemical formulations of molecular structure allow for the possibility of such a view of molecules? Although we do not take supervenience as self-evidently true, nor do we deny that it is questionable whether and to what extent it can be maintained when considering for example the mind-body relation (p. 96, Dupré 1993), within the context of chemistry and quantum mechanics it is safe to argue that the rejection of supervenience would lead to the acceptance of a thesis that is scientifically dubious. Firstly, there is no positive scientific evidence that molecules behave in a manner that is totally independent from the perspective of quantum mechanics; rather the contrary. The only evidence that has been presented is negative [for example Hendry (2006b) and Hendry (2010)] in the sense of presenting specific and unique cases of chemical properties whose changes have not (supposedly) been epistemically recovered in the quantum mechanical description. Even if, for example, we accept that isomers cannot be differentiated quantum mechanically with respect to some of their structural properties, changes to the majority of chemical properties (whether structural or not) are in fact reflected in changes in the wave function of the respective system. The fact that only some structural changes may not be described quantum mechanically should be regarded as evidence in favour of supervenience rather than as evidence against it, especially if one considers the array of alternative epistemic justifications that have been provided in order to explain such 'failures' of the quantum formalism [see for example Scerri (2012)]. 
The fourth criterion of Kincaid's model is focused on exactly this point (i.e. (4)). It requires that there is sufficient scientific evidence that could inductively support that higher level properties supervene upon lower level ones.

(4) It is possible to inductively support supervenience by citing examples of quantum mechanical mechanisms that fix or realise higher level properties (p. 66, Kincaid 1997)

The support of supervenience can be strengthened by citing examples of mechanisms that illustrate how the chemical properties are fixed or determined by the properties of lowerlevel entities. Kincaid does not require that there is a complete set of mechanisms in the quantum description that realise or bring about the complete set of higher-level properties; he merely wishes to support supervenience by pointing out that there exist examples of mechanisms that illustrate his point. The most striking perhaps quantum mechanism is the one used to define the orbital structure of atoms in chemistry.

This mechanism is based on Schrödinger's specification of the three quantum numbers and on Pauli's exclusion principle that specifies the fourth quantum number. It outlines the building rules with which chemists are able to specify the electron configuration of atoms, and consequently it provides the basis for specifying what kind of bonds different types of atoms can form, and with what type of atoms that could be.

The electron configuration of an atom is a "list of all its occupied orbitals, with the number of electrons that each one contains" (p. 33, Atkins and Jones 2010). This list is completed by following certain building rules that are based on Schrödinger's solution of the wave function for the hydrogen atom, and on Pauli's Principle. Specifically, atomic orbitals refer to one-electron orbital eigenfunctions which are "based on the attraction of the nucleus for the electron we are considering plus the average repulsion of all the other electrons" (p. 13, Mulliken 1967). The shape and size of the area that the electron is most probably occupying around the nucleus is designated by the four quantum numbers.

The first quantum number is the principle quantum number, ${ }^{14}$ which represents the energy and size of the atomic shell. This classification of orbitals into atomic shells is further sub-divided because an electron can occupy an atomic shell by orbiting around in various forms. These different forms are captured by the notion of subshell, and each subshell is represented by the angular momentum quantum number, 1. In chemistry, one writes down the shell and subshell that an electron occupies, in terms of the value of the principle quantum number (n) and the letters s,p,d,f that correspond to the values of the angular momentum quantum number (1). So, for example $1 \mathrm{~s}$ represents a subshell of the $\mathrm{s}$-form that occupies the lowest atomic shell, $2 \mathrm{p}$ represents the $\mathrm{p}$ subshell in the second atomic shell etc.

Moreover, from the quantum calculation of the probability density of finding an electron at a particular subshell, it turns out that not all atomic shells allow all forms of subshells (p. 33, Atkins and Jones 2010). This is because for example, the wave function of a p-electron vanishes at its nucleus. That is why, in shell-1 an electron will be found to occupy only an s-subshell, and not a p- or f-subshell. Keeping this in mind, quantum mechanics has posited a ranking of orbitals of the following form:

$$
1 \mathrm{~s}<2 \mathrm{~s}<2 \mathrm{p}<3 \mathrm{~s}<3 \mathrm{p}<3 \mathrm{~d}<\cdots
$$

\footnotetext{
${ }^{14}$ I follow the technical notation of Atkins and Friedman (2005) and Atkins and Jones (2010).
} 
The third feature required to specify the orbital of an electron is the magnetic quantum number, $m_{1}$. The value of $m_{1}$ specifies the individual orbitals within a subshell and represents the different direction of each orbital within that subshell.

The last element for a complete description of the electrons' orbitals, is derived from Pauli's Principle. Pauli introduced a rule according to which a maximum of two electrons can occupy one orbital; namely an orbital with a specific set of $\left(n, 1, m_{1}\right)$ numbers. These two electrons are viewed as if moving on opposite directions on the orbital and they are represented uniquely in chemistry by one more quantum number, namely spin (which takes two possible values; $+1 / 2,-1 / 2$ ).

In sum, the possible values of the three quantum numbers, together with their subshell ranking and the Pauli Principle, act as building rules that chemists use in order to write the electron configuration of an atom. By following those rules, it is possible to represent the orbitals that the electrons of a particular atom will occupy in terms of them having particular size, shape and orientation. So, for example, the hydrogen atom has one electron. It must be the case that the lowest energy shells are occupied first; so hydrogen's electron occupies shell-1. Also, we know that in shell-1 the electron moves necessarily in an s-subshell, namely spherically around the nucleus. So, the electron of a hydrogen atom occupies orbital $1 \mathrm{~s}$. For the carbon atom, which has six electrons, two electrons will occupy shell-1 on an s-subshell with opposite spin (due to Pauli's Principle), two more will occupy shell-2 on an s-subshell, and the other two will occupy shell-2 on a p-subshell. Which orbital must be filled first is indicated by the aforementioned ranking of orbitals $(1 \mathrm{~s}<2 \mathrm{~s}<2 \mathrm{p}<3 \mathrm{~s}<3 \mathrm{p}<3 \mathrm{~d}<\cdots)$.

This mechanism illustrates how certain chemical properties of atoms and molecules supervene on quantum properties of electrons and nuclei. The number of electrons that fill the last atomic orbital and the particular quantum characteristics of the atomic orbital (in terms of the quantum numbers that specify it), realise the overall structure of the atom, and subsequently its chemical behaviour. This is particularly important for the chemical description of atoms and molecules since, specifying the structure of each atom that participates in the formation of a molecule, defines and explains the particular bonds that are formed with other atoms within the molecule as well as those bonds' properties, and thus contribute to the explanation and prediction of the structure and reactivity of the molecule.

(5) Both chemistry and quantum mechanics heuristically depend on each other (p. 66, Kincaid 1997)

For the two theories to be considered as 'heuristically dependent' upon each other, the paper takes that it should be the case that both quantum mechanics and chemistry;

i. have enriched or influenced the research questions that concern the other discipline

ii. have developed tools, methods or models that have accommodated the research questions of the other discipline.

Indeed, the relation of chemistry and quantum mechanics has illustrated such a heuristic interdependence and has enriched the research questions of both theories. Quantum mechanics led chemists to further investigate the structure of atoms and to incorporate in the chemical description quantum mechanical postulations about the structure of atoms. Conceptual issues such as the nature of the chemical bond still maintain a lively debate among chemists and physicists, since quantum mechanics revealed the existence of factors that affect the conceptual understanding of basic chemical concepts. On the other hand, chemistry also raised questions that enriched quantum mechanical research; different 
quantum models are continuously being developed in order to expand the quantum mechanical description to various kinds of entities (i.e. atoms, diatomic molecules, polyatomic molecules, metals etc.).

Moreover, the history of quantum chemistry reveals a continuous exchange of tools, methods and models in the effort to satisfy explanatory and predictive needs of both sides. Chemists have gained a substantial amount of quantitative and qualitative information concerning molecular structure by quantum models that solve, via approximations and idealisations, the Schrödinger equation for different types of atoms or molecules. Certain techniques manage to give us accurate descriptions of small molecules, whereas others are more successful in describing larger (polyatomic) molecules, or types of matter such as metals (see Hoffman 1990). Moreover, through the development of the Molecular Orbital approach, quantum chemists developed novel visual representations of molecular orbitals that enriched chemistry's understanding of molecular structure in organic chemistry.

All in all, exploring the history of the development of chemistry, quantum mechanics and computation illustrates how those disciplines of scientific investigation have utilised each others' models in order to expand their research and to accommodate their explanatory, heuristic and predictive needs (see Gavroglu and Simoes 2012). Currently, this interdependence between chemistry and quantum mechanics has been extended in a wide range of sub-disciplines, where quantum mechanical models are used in material science, drug design etc. (p. 245, Matta 2013). Although those needs may be different for each discipline, this does not mean that the models they use are not provided by other subdisciplines. In this sense, Kincaid's criterion is fulfilled.

(6) Chemistry and quantum mechanics confirmationally depend on each other (p. 66, Kincaid 1997)

Chemistry and quantum mechanics are confirmationally dependent in two ways. Firstly, the results of chemical experimentation have played a vital role both in the development and in the evaluation of quantum models. The quantitative results of quantum models are compared with experimental results so as to see how accurate the model is and to what direction changes need to be made within the model so as to minimise error. Those experimental results are provided through the work of experimental chemists who, with the use of spectroscopical and other techniques, calculate chemical properties. This aspect of confirmational interdependence is illusttrated in (Weisberg 2008), who evaluates the predictive success of different quantum models by comparing the calculation of the dissociation energy and the equilibrium distance of atomic nuclei in quantum models, with the respective results of chemists' experimental calculation of the dissociation energy and bond length.

Secondly, novel predictions of chemical phenomena have been made with the help of quantum mechanics. For example, the specification of atomic structure by quantum mechanics helped chemists understand spectroscopic results and thus predict novel elements, in accordance to the periodic table (p. 213, Needham 2004). Moreover, quantum models have made novel predictions about, among others, pericyclic reactions (p. 1057, Hendry 2004), large molecules, and metals, enriching thus chemical knowledge and corroborating chemistry's theoretical postulations.

(7) Chemistry and quantum mechanics contribute in the explanatory endeavours of each other (p. 66, Kincaid 1997)

Kincaid does not specify in detail what he means by 'explanation' nor does he explicitly support a particular philosophical model of explanation (i.e. deductive nomological, SR 
model, causal mechanical etc.). The paper retains this neutral position and understands explanation in a naive, qualitative and scientistic sense. Specifically, it takes as explanatory any narrative developed within the scientific literature and pedagogy, and employed by working scientists in order to understand the phenomena which they examine.

In the context of such an understanding of 'explanation', the paper takes that Kincaid's seventh criterion holds with respect to chemistry and quantum mechanics. Specifically, advances in quantum mechanics have enriched chemical explanations of molecular structure and at times even led to their revision. This can be argued from an opposite perspective as well; chemical explanations of molecular structure have been used in the development of quantum models and have guided quantum chemists towards a more accurate interpretation of the mathematical description of atoms and molecules (as this manifests via the Schrödinger equation). In fact, genuine explanatory differences both between different quantum models, and between quantum models and chemistry have raised heated discussions in order to consolidate or review the explanatory account of one or both of the sides.

There are several examples that illustrate this interdependence, among which are the following;

- The use of the quantum mechanical account of atomic structure in the explanation of the periodic table. As already illustrated, quantum mechanics provide chemists a mechanism to specify and explain atomic structure in terms of electron configuration. This proved particularly helpful in the explanation of the periodic table and of its success in classifying chemical elements according to similar chemical and physical properties (Scerri 2006). Despite the fact that the periodic table was constructed long before the advent of quantum mechanics, quantum mechanics provides the explanation of why the chemical elements belonging in specific vertical and horizontal columns exhibit particular similarities.

- Quantum mechanics has enriched the understanding of molecular structure through the explanatory conclusions derived by different quantum models that attempt to describe molecular structure. For example, the development of the molecular orbital (MO) approach in quantum mechanics has revealed the effect of electron delocalisation on the overall stability of a molecule. Modern versions of the MO approach, like the Hartree-Fock methods and the Configuration Interaction approach (CI), take into account the repulsion of electrons, the ionic character of chemical bonds and the mixing of higher energy states, revealing additional factors that enrich chemistry's understanding of basic chemical concepts pertained to molecular structure (Weisberg 2008).

- Quantum mechanical models have been developed with the help of theoretical chemistry in the sense that the models are calibrated or corrected through the use of chemical postulations or assumptions from chemistry (p. 183, Hendry 2010).

Concluding this section, there is sufficient evidence that supports the heuristic, confirmatory and explanatory interdependence of quantum mechanics and chemistry. This epistemic interdependence, together with the satisfaction of supervenience and the logical compatibility of the two theories, define the specific model of unification and, consequently, strongly support that quantum mechanics and chemistry are indeed unified in such a way. 


\section{Concluding remarks: the virtues of Kincaid's unificatory model}

Kincaid's unificatory model is primarily an epistemic position regarding how different disciplines formulate a unified body of knowledge that is continuously influenced and developed through each discipline's explanatory, confirmatory and heuristic advances. Molecular structure is described, explained and predicted through the theoretical postulations and conceptions, but also the experimental and representational tools of both quantum mechanics and chemistry, resulting in the formation of a unified body of knowledge.

This understanding of unification is compatible with various epistemic and ontological positions about chemistry and quantum mechanics. Although an enumeration of all the alternatives is not currently possible, let me provide some examples. On the one hand, it is possible to maintain the autonomy of the two disciplines, if one understands the term as merely signifying the existence of distinct research groups with distinct research goals, that employ independent methods, tools, models, or even conceptions. On the other hand, the model is also compatible with the existence of local reductions between the two descriptions; namely Kincaid's unity is not refuted if, for example, certain properties of the higher level description are connected via bridge laws to sets of lower level properties. Concerning metaphysical issues, the supervenience thesis does not necessarily entail that the higher level entities and properties are eliminable from reality (though this is also a compatible position). One could still maintain that molecules exist and that their structure is real, without contradicting Kincaid. These points however will be discussed on another occasion.

Open Access This article is distributed under the terms of the Creative Commons Attribution 4.0 International License (http://creativecommons.org/licenses/by/4.0/), which permits unrestricted use, distribution, and reproduction in any medium, provided you give appropriate credit to the original author(s) and the source, provide a link to the Creative Commons license, and indicate if changes were made.

\section{References}

Atkins, P., Friedman, R.: Molecular Quantum Mechanics. Oxford University Press, Oxford (2005)

Atkins, P., Jones, L.: Chemical Principles-The Quest for Insight, 5th edn. W.H. Freeman and Company, New York (2010)

Bokulich, A.: Reexamining the Quantum-Classical Relation. Cambridge University Press, Cambridge (2008)

Chang, H.: Is Water H2O? Evidence, Realism and Pluralism, Boston Studies in the Philosophy and history of Science, vol. 293. Springer, Dordrecht (2012)

Curd, M., Cover, J.A. (eds.): Philosophy of Science-The Central Issues. W.W. Norton \& Company, Inc., New York (1998)

Dizadji-Bahmani, F., Frigg, R., Hartmann, S.: Who's Afraid of Nagelian Reduction? Erkenntnis (1975-), 73(3), Reduction and The Special Sciences, pp. 393-412, Springer (2010)

Dupré, J.: The Disorder of Things. Harvard University Press, Cambridge (1993)

Gavroglu, K., Simoes, A.: Neither Physics nor Chemistry: A History of Quantum Chemistry. The MIT Press, Cambridge (2012)

Hendry, R.F.: The physicists, the chemists, and the pragmatics of explanation. Philos. Sci. 71(5), 1048-1059 (2004)

Hendry, R.F.: Two conceptions of the chemical bond. Philos. Sci. 75(5), 909-920 (2006)

Hendry R.F.: Is there Downward Causation in Chemistry?, Philosophy of Chemistry, Vol 242 of the series Boston Studies in the Philosophy of Science, pp. 173-189 (2006b)

Hendry, R.F.: Ontological reduction and molecular structure. Stud. Hist. Philos. Mod. Phys. 41, $183-191$ (2010) 
Hendry, R.F., Woody, A.I., Needham, P.: Volume 6: Philosophy of Chemistry, eds. Handbook of the philosophy of science. Elsevier, Amsterdam (2012)

Hettema, H.: Reducing Chemistry to Physics. Limits, Models, Consequences. Rijksuniversiteit Groningen, Groningen (2012)

Hettema, H.: Linking chemistry with physics: a reply to Lombardi. Found. Chem. 16, 193-200 (2014)

Hofmann, J.R.: How the models of chemistry vie. In: PSA: Proceedings of the Biennial Meeting of the Philosophy of Science Association, vol. 1990, Volume One: Contributed Papers, pp. $405-419$ (1990)

Kellert, S.H., Longino, H.E., Waters, C.K. (eds): Volume XIX Scientific Pluralism. In: Minnesota Studies in the Philosophy of Science. University of Minnesota Press (2006)

Kincaid, H.: Individualism and the Unity of Science. Rowman \& Littlefield Publishers Inc, Maryland (1997)

Ladyman, J., Ross, D.: Every Thing Must Go. OUP, Oxford (2007)

Le Poidevin, R.: Missing elements and missing premises: a combinatorial argument for the ontological reduction of chemistry. Br. J. Philos. Sci. 56, 117-134 (2005)

Lombardi, O., Labarca, M.: The ontological autonomy of the chemical world. Found. Chem. 7, 125-148 (2005)

Lombardi, O.: Linking chemistry to physics: arguments and counterarguments. Found. Chem. 16, 181-192 (2014)

Matta, C.F.: Special issue: philosophical aspects and implications of the quantum theory of atoms in molecules (QTAIM). Found. Chem. 15, 245-251 (2013)

Mulliken, R.S.: Spectroscopy, molecular orbitals, and chemical bonding. Science New Series 157, 13-24 (1967)

Needham, P.: When did atoms begin to do any explanatory work in chemistry? Int. Stud. Philos. Sci. 18(2-3), 199-219 (2004)

Needham, P.: Nagel's analysis of reduction: comments in defence as well as critique. Stud. Hist. Philos. Mod. Phys. 41, 163-170 (2010)

Orilia, F., Swoyer, C.: Properties. In The Stanford Encyclopedia of Philosophy (2016)

Pullman, B.: The Atom in the History of Human Thought. Oxford University Press, Oxford (1998)

Ramsey, J.L.: Molecular shape, reduction, explanation and approximate concepts. Synthese 111, 233-251 (1997)

Scerri, E.: The Periodic Table, Its Story and Its Significance. Oxford University Press, Oxford (2006)

Scerri, E.: Top-down causation regarding the chemistry-physics interface: a sceptical view. Interface Focus 2, 20-25 (2012)

Scerri, E., Fisher, G. (eds.): Essays in the Philosophy of Chemistry. Oxford University Press, Oxford (2016)

Schummer, J.: The methodological pluralism of chemistry and its philosophical implications. In: Scerri, E., McIntyre, L. (eds.) Philosophy of Chemistry: Growth of a New Discipline, pp. 57-72. Springer, Dordrecht (2014)

Weisberg, M.: Challenges to the structural conceptions of chemical bonding. Philos. Sci. 75, 932-946 (2008) 